\title{
On explicit inversion of a subclass of operators with $D$-difference kernels and Weyl theory of the corresponding canonical systems
}

\author{
A.L. Sakhnovich, A.A. Karelin, J. Seck-Tuoh-Mora, \\ G. Perez-Lechuga, M. Gonzalez-Hernandez
}

\begin{abstract}
Explicit inversion formulas for a subclass of integral operators with $D$-difference kernels on a finite interval are obtained. A case of the positive operators is treated in greater detail. An application to the inverse problem to recover canonical system from a Weyl function is given.
\end{abstract}

MSC(2000) Primary 34A55, 45Q05; Secondary 47B65, 47G10

Keywords: integral operator with difference kernel, operator with Ddifference kernel, explicit inversion, canonical system, inverse problem, Weyl function.

\section{Introduction}

Integral operators with difference kernels are important in mathematics and applications and are actively used in the study of numerous homogenious processes. The papers $[13,18]$ on the inversion of the operators with difference kernels on the semi-axis became classical. Various results and references on the operators with difference kernels on a finite interval or a system of intervals are given in $[24,26]$. Interesting explicit results on the inversion of the operators with exponential type difference kernels on a finite interval one can find in $[2,12]$. 
Operators with $D$-difference kernels in $L_{p}^{2}(0, l)$, which we shall treat, are bounded operators of the form

$$
\begin{aligned}
& S_{l} f=S f=\frac{d}{d x} \int_{0}^{l} s(x, t) f(t) d t, \quad s(x, t)=\left\{s_{i j}(x, t)\right\}_{i, j=1}^{p}, \\
& s_{i j}(x, t)=s_{i j}\left(d_{i} x-d_{j} t\right), \quad s_{i j}(x) \in L^{2}\left(-d_{j} l, d_{i} l\right),
\end{aligned}
$$

where $D=D^{*}=\operatorname{diag}\left\{d_{1}, d_{2}, \ldots, d_{p}\right\}>0$ is a fixed $p \times p$ diagonal matrix. The notion of an operator with a $D$-difference kernel is a natural generalization of the operator with a difference kernel, i.e., of the case $D=I_{p}$, where $I_{p}$ is the $p \times p$ identity matrix. The class of operators with $D$-difference kernels on a finite interval includes the operators with difference kernels on systems of intervals, which are important, for instance, in elasticity theory, diffraction theory, and the theory of stable processes (see [16] and Chapter 6 in [26]).

Explicit inversion formulas for an interesting subclass of operators with $D$-difference kernels are obtained in Section 2 of this paper using the classical results on semiseparable operators. Note also that the inversion of semiseparable matrices and operators is another interesting and actively developed theory, see [8, 9] and bibliography in [30]. Some further possible applications are connected with the paper [17].

Operator identities for the operators with $D$-difference kernels are discussed in Section 3.

The case of positive and boundedly invertible operators with $D$-difference kernels is treated further in Theorem 4.3 of Section 4. As an application, we solve explicitly in terms of Weyl functions an inverse problem for a subclass of canonical systems. Some results from [28, 29] are developed further in this section too.

We use the standard notations $\mathbb{C}$ and $\mathbb{C}_{+}$for the complex plane and upper semi-plane, respectively. By $\left\{\mathcal{H}_{1}, \mathcal{H}_{2}\right\}$ we denote the class of the bounded linear operators acting from $\mathcal{H}_{1}$ into $\mathcal{H}_{2}$, and by $\sigma(\beta)$ we denote the spectrum of $\beta$. 


\section{Inversion of operators with $D$-difference ker- nels}

Consider a self-adjoint operator with $D$-difference kernel

$$
S=I+\int_{0}^{l} k(x, t) \cdot d t, \quad k(x, t)=\left\{k_{i j}(x, t)\right\}_{i, j=1}^{p}=k_{i j}\left(d_{i} x-d_{j} t\right),
$$

where $I$ is the identity operator, the $p \times p$ matrix function $k(x)$ on the right hand side of the second relation in (2.1) is given by the equalities

$$
k(x)=\Theta_{2}^{*} e^{i x \beta^{*}} \Theta_{1} \quad(x>0), \quad k(-x)=k(x)^{*},
$$

$\Theta_{m}(m=1,2)$ is an $n \times p$ matrix, and $\beta$ is an $n \times n$ matrix for some integer $n>0$. Without loss of generality we assume further that

$$
d_{1} \geq d_{2} \geq \ldots \geq d_{p}>0 .
$$

Remark 2.1 We suppose that equalities (2.2) hold on $\left(0, d_{1} l\right)$, and so, according to (2.3), each entry $k_{i j}(x)$ is determined by (2.2) on the interval, which contains $\left(-d_{j} l, d_{i} l\right)$, i.e., the operator $S$ of the form (2.1) is determined by (2.2).

Introduce the operator

$$
E \in\left\{L_{p}^{2}(0, l), L^{2}(D)\right\} \quad L^{2}(D):=L^{2}\left(0, d_{1} l\right) \oplus L^{2}\left(0, d_{2} l\right) \oplus \ldots \oplus L^{2}\left(0, d_{p} l\right)
$$

by the equality $(E f)_{j}(z)=f_{j}\left(z / d_{j}\right)$. We shall denote also by $E$ the corresponding operator from $\left\{L_{p}^{2}(0, l), L_{p}^{2}\left(0, d_{1} l\right)\right\}$ with the natural embedding of $L^{2}(D)$ into $L_{p}^{2}\left(0, d_{1} l\right)$ :

$$
(E f)_{j}(z)=f_{j}\left(z / d_{j}\right) \quad\left(0<z<d_{j} l\right), \quad(E f)_{j}(z)=0 \quad\left(d_{j} l<z<d_{1} l\right) .
$$

By (2.1) and (2.5) it is easy to see that

$$
\begin{aligned}
& S=E^{-1}\left(I+\int_{0}^{a} \widetilde{k}(y, z) \cdot d z\right) E, \quad a:=d_{1} l \\
& \widetilde{k}(y, z)=\left\{\widetilde{k}_{i j}(y, z)\right\}_{i, j=1}^{p}, \quad \widetilde{k}_{i j}(y, z)=0 \quad \text { if } z>d_{j} l \text { or } y>d_{i} l \\
& \widetilde{k}_{i j}(y, z)=\frac{1}{d_{j}} k_{i j}(y-z) \quad \text { if } 0<z<d_{j} l \text { and } 0<y<d_{i} l
\end{aligned}
$$


According to (2.2), (2.7), and (2.8), the operator

$$
\widetilde{S}=I+\int_{0}^{a} \widetilde{k}(y, z) \cdot d z
$$

is not an operator with a difference kernel but it is a semiseparable operator. Recall [8] that the integral operator $\widetilde{S}$ of the form (2.9) is called semiseparable, when $\widetilde{k}$ admits representation

$$
\widetilde{k}(y, z)=F_{1}(y) G_{1}(z) \quad \text { for } y>z, \quad \widetilde{k}(y, z)=F_{2}(y) G_{2}(z) \quad \text { for } y<z,
$$

where $F_{1}$ and $F_{2}$ are $p \times n$ matrix functions and $G_{1}$ and $G_{2}$ are $n \times p$ matrix functions for some $n>0$. It is assumed that the entries of $F_{1}, F_{2}, G_{1}$, and $G_{2}$ are square integrable. When the operator $\widetilde{S}$ is invertible and its kernel $\widetilde{k}$ is given by (2.10), the kernel of the operator $\widetilde{T}=\widetilde{S}^{-1}$ is expressed in terms of the $2 n \times 2 n$ solution $U$ of the differential equation

$$
\left(\frac{d}{d y} U\right)(y)=\widetilde{J} \widetilde{H}(y) U(y), \quad y \geq 0, \quad U(0)=I_{2 n}
$$

where

$$
\begin{aligned}
& \widetilde{J} \widetilde{H}(y):=B(y) C(y), \quad \widetilde{J}=\left(\widetilde{J}^{*}\right)^{-1}=\left[\begin{array}{rr}
0 & -I_{p} \\
I_{p} & 0
\end{array}\right] . \\
& B(y)=\left[\begin{array}{c}
-G_{1}(y) \\
G_{2}(y)
\end{array}\right], \quad C(y)=\left[\begin{array}{ll}
F_{1}(y) & F_{2}(y)
\end{array}\right] .
\end{aligned}
$$

Namely, we have (see, for instance, [8])

$$
\begin{gathered}
\widetilde{T}=\widetilde{S}^{-1}=I+\int_{0}^{a} \widetilde{T}(y, z) \cdot d z, \\
\widetilde{T}(y, z)=\left\{\begin{array}{l}
C(y) U(y)\left(I_{2 n}-P^{\times}\right) U(z)^{-1} B(z), \quad y>z, \\
-C(y) U(y) P^{\times} U(z)^{-1} B(z), \quad y<z .
\end{array}\right.
\end{gathered}
$$

Here $P^{\times}$is given in terms of the $n \times n$ blocks $U_{21}(a)$ and $U_{22}(a)$ of $U(a)$ :

$$
P^{\times}=\left[\begin{array}{lc}
0 & 0 \\
U_{22}(a)^{-1} U_{21}(a) & I_{n}
\end{array}\right],
$$


and the invertibility of $U_{22}(a)$ is a necessary and sufficient condition for the invertibility of $\widetilde{S}$.

When the semiseparable operator $\widetilde{S}$ is not invertible, its kernel subspace is given by the equality ([8], p. 157):

$$
\text { Ker } \widetilde{S}=\left\{h(y): h(y)=C(y) U(y)\left[\begin{array}{l}
0 \\
g
\end{array}\right], U_{22}(a) g=0\right\} .
$$

Rewrite $D$ in the form

$$
\begin{aligned}
& D=\operatorname{diag}\left\{\widetilde{d}_{1} I_{p_{1}}, \ldots, \widetilde{d}_{k} I_{p_{k}}\right\}, \quad p_{1}+\ldots+p_{k}=p \\
& \widetilde{d}_{j_{1}}>\widetilde{d}_{j_{2}}>0 \quad\left(j_{1}<j_{2} \leq k\right)
\end{aligned}
$$

and put

$$
\widetilde{d}_{k+1}=0, \quad P_{k+1}=I_{p}, \quad P_{j}=\operatorname{diag}\left\{I_{p_{1}}, \ldots, I_{p_{j-1}}, 0, \ldots, 0\right\}(2 \leq j \leq k) .
$$

Then, in view of $(2.2),(2.7),(2.8)$, and (2.13) we have

$$
B(y)=e^{-y \mathcal{A}}\left[\begin{array}{c}
-\Theta_{1} \\
\Theta_{2}
\end{array}\right] D^{-1} P_{j}, \quad C(y)=P_{j}\left[\begin{array}{cc}
\Theta_{2}^{*} & \Theta_{1}^{*}
\end{array}\right] e^{y \mathcal{A}}
$$

for

$$
\widetilde{d}_{j} l<y<\widetilde{d}_{j-1} l \quad(2 \leq j \leq k+1), \quad \mathcal{A}:=i\left[\begin{array}{cc}
\beta^{*} & 0 \\
0 & \beta
\end{array}\right] .
$$

Remark 2.2 By (2.5), (2.17) and (2.20), it is immediate that

$$
\operatorname{Ker} \widetilde{S} \in \operatorname{Im} E \text {. }
$$

where Im means image. The integral parts of $S$ and $\widetilde{S}$ are compact operators. Hence, if $\widetilde{S}$ is not invertible, then Ker $\widetilde{S} \neq 0$, and according to (2.22) the subspace $E^{-1} \operatorname{Ker} \widetilde{S}$ is well defined. In view of (2.6) and (2.9), we have $S E^{-1}$ Ker $\widetilde{S}=0$, i.e., $S$ is not invertible too. It follows from (2.6), (2.14), and (2.15) that if $\widetilde{S}$ is invertible, then $S$ is invertible. In other words, $S$ and $\widetilde{S}$ are simultaneousy invertible. 
Next, introduce notations

$$
\mathcal{A}_{j}^{\times}=\mathcal{A}+Y_{j}, \quad Y_{j}=\left[\begin{array}{c}
-\Theta_{1} \\
\Theta_{2}
\end{array}\right] D^{-1} P_{j}\left[\begin{array}{ll}
\Theta_{2}^{*} & \Theta_{1}^{*}
\end{array}\right] .
$$

For $2 \leq j \leq k+1$, put

$$
U(y)=e^{-y \mathcal{A}} e^{\left(y-\widetilde{d}_{j} l\right) \mathcal{A}_{j}^{\times}} e^{\widetilde{d}_{j} l \mathcal{A}} U\left(\widetilde{d}_{j} l\right) \quad\left(\widetilde{d}_{j} l \leq y \leq \widetilde{d}_{j-1} l\right), \quad U(0)=I_{2 n},
$$

Now, we are prepared to formulate the inversion theorem.

Theorem 2.3 Let $S$ be an operator with the D-difference kernel, which has the form (2.1), where $k$ is given by (2.2) and D satisfies (2.18). Let also $\operatorname{det} U_{22}(a) \neq 0$ for $U$ given by (2.24). Then $S$ is invertible and its inverse is given by the formula $S^{-1}=E^{-1} \widetilde{T} E$, where $E$ is defined by (2.5) and $\widetilde{T}$ is given by (2.14)-(2.16). The matrix functions $B$ and $C$ in (2.15) are given by (2.20) and the $\widetilde{J}$-unitary matrix function $U$ in (2.15) has the form (2.24).

Proof. To prove the theorem we need to show that $U$ of the form (2.24) satisfies (2.11). Then by the properties of the semiseparable operators we shall obtain that $\widetilde{S}$ given by (2.9) is invertible and that $\widetilde{T}=\widetilde{S}^{-1}$ is given by (2.14)-(2.16), (2.24). The formula $S^{-1}=E^{-1} \widetilde{T} E$ will be immediate from (2.6).

By formulas (2.20) and (2.23) it is easy to see that $U$ of the form (2.24) satisfies equation

$$
\begin{aligned}
& \left(\frac{d}{d y} U\right)(y)=e^{-y \mathcal{A}}\left(\mathcal{A}_{j}^{\times}-\mathcal{A}\right) e^{y \mathcal{A}} e^{-y \mathcal{A}} e^{\left(y-\widetilde{d}_{j} l\right) \mathcal{A}_{j}^{\times}} e^{\widetilde{d}_{j} l \mathcal{A}} U\left(\widetilde{d}_{j} l\right) \\
& =e^{-y \mathcal{A}} Y_{j} e^{y \mathcal{A}} U(y)=B(y) C(y) U(y)
\end{aligned}
$$

for $0 \leq y \leq a$. Hence, by (2.12) $U$ satisfies (2.11).

Finally, let us prove that $U$ is $\widetilde{J}$-unitary, i.e., $U(y)^{*} \widetilde{J} U(y)=\widetilde{J}$. Indeed, according (2.21) we have

$$
\mathcal{A}^{*}=-\widetilde{J} \mathcal{A} \widetilde{J}^{*}
$$

As we noted in (2.25), the equality $B(y) C(y)=e^{-y \mathcal{A}} Y_{j} e^{y \mathcal{A}}$ is true. Thus, taking into account (2.12), (2.23), and (2.26) we obtain

$$
\begin{aligned}
& \widetilde{H}(y)=\widetilde{J}^{*} e^{-y \mathcal{A}} Y_{j} e^{y \mathcal{A}}=e^{y \mathcal{A}^{*}} \widetilde{J}^{*} Y_{j} e^{y \mathcal{A}} \\
& =e^{y \mathcal{A}^{*}}\left[\begin{array}{c}
\Theta_{2} \\
\Theta_{1}
\end{array}\right] D^{-1} P_{j}\left[\begin{array}{ll}
\Theta_{2}^{*} & \Theta_{1}^{*}
\end{array}\right] e^{y \mathcal{A}} \geq 0 .
\end{aligned}
$$


It follows from $(2.27)$ that $\widetilde{H}^{*}=\widetilde{H}$. Therefore, formulas $(2.11)$ and $(2.12)$ imply $\frac{d}{d y}\left(U(y)^{*} \widetilde{J} U(y)\right)=0$. Moreover, from $\frac{d}{d y}\left(U(y)^{*} \widetilde{J} U(y)\right)=0$ and $U(0)=I_{2 n}$ we get $U(y)^{*} \widetilde{J} U(y)=\widetilde{J}$.

Remark 2.4 If $S$ is invertible, then from Theorem 2.3 we derive

$$
T=S^{-1}=I+\int_{0}^{l}\left\{T_{i j}(x, t)\right\}_{i, j=1}^{p} \cdot d t
$$

where for $d_{i} x>d_{j}$ t and $e_{i}=\left[\begin{array}{llllll}\overbrace{0} \ldots \ldots & 0 & 1 & 0 & \ldots & 0\end{array}\right]$ we have

$$
T_{i j}(x, t)=e_{i}\left[\begin{array}{ll}
\Theta_{2}^{*} & \Theta_{1}^{*}
\end{array}\right] e^{d_{i} x \mathcal{A}} U\left(d_{i} x\right)\left(I_{2 n}-P^{\times}\right) U\left(d_{j} t\right)^{-1} e^{-d_{j} t \mathcal{A}}\left[\begin{array}{c}
-\Theta_{1} \\
\Theta_{2}
\end{array}\right] e_{j}^{*},
$$

and for $d_{i} x<d_{j}$ t we have

$$
T_{i j}(x, t)=-e_{i}\left[\begin{array}{ll}
\Theta_{2}^{*} & \Theta_{1}^{*}
\end{array}\right] e^{d_{i} x \mathcal{A}} U\left(d_{i} x\right) P^{\times} U\left(d_{j} t\right)^{-1} e^{-d_{j} t \mathcal{A}}\left[\begin{array}{c}
-\Theta_{1} \\
\Theta_{2}
\end{array}\right] e_{j}^{*} .
$$

\section{Operator identities for operators with $D$ - difference kernels}

According to [26] (Ch. 6) a bounded in $L_{p}^{2}(0, l)$ operator $S$ with $D$-difference kernel, that is, an operator of the form (1.1), (1.2) satisfies the operator identity

$$
A S-S A^{*}=i \Pi J \Pi^{*},
$$

where $A_{l}=A \in\left\{L_{p}^{2}(0, l), L_{p}^{2}(0, l)\right\}, \Pi_{l}=\Pi=\left[\begin{array}{ll}\Phi_{1} & \Phi_{2}\end{array}\right], \Phi_{k} \in\left\{\mathbb{C}^{p}, L_{p}^{2}(0, l)\right\}$, the index "l" is often omitted in our notations, and

$$
A=i D \int_{0}^{x} \cdot d t, \quad \Phi_{1} g=D s(x, 0) g, \quad \Phi_{2} g \equiv g
$$

It is said that $A, S$, and $\Pi$, which satisfy (3.1), form an $S$-node. Further we assume that $A$ and $\Phi_{2}$ have the form (3.2). Operator identities play an important role in the study of structured operators [26, 27, 29]. 
Let us show that not only the operator with the $D$-difference kernel satisfies (3.1) but the inverse statement is also true, i.e., (3.1) implies that $S$ is an operator with a $D$-difference kernel (see also the corresponding statement in Example 1.2, p. 104 [29]). Quite similar to the proof of Theorem 1.3 ([26], p. 11), where the case $D=I_{p}$ was treated, one can prove the following theorem

Theorem 3.1 Suppose a bounded operator $T \in\left\{L_{p}^{2}(0, l), L_{p}^{2}(0, l)\right\}$ satisfies the operator identity

$$
\begin{aligned}
T A-A^{*} T & =i \int_{0}^{l} Q(x, t) \cdot d t, \\
Q(x, t) & =Q_{1}(x) Q_{2}(t),
\end{aligned}
$$

where $Q, Q_{1}$, and $Q_{2}$ are $p \times p, p \times \widehat{p}$, and $\widehat{p} \times p(\widehat{p}>0)$ matrix-functions, respectively. Then $T$ has the form

$$
T f=\frac{d}{d x} \int_{0}^{l} \frac{\partial}{\partial t} \Upsilon(x, t) f(t) d t,
$$

where $\Upsilon(x, t)=\left\{\Upsilon_{i j}(x, t)\right\}_{i, j=1}^{p}$ is absolutely continuous in $t$, and

$$
\begin{aligned}
\Upsilon_{i j}(x, t):= & \left(2 d_{i} d_{j}\right)^{-1} \int_{d_{i} x+d_{j} t}^{f_{\text {min }}} Q\left(\frac{u+d_{i} x-d_{j} t}{2 d_{i}}, \frac{u-d_{i} x+d_{j} t}{2 d_{j}}\right) d u, \\
& f_{\text {min }}:=\min \left(d_{i}(2 l-x)+d_{j} t, d_{i} x+d_{j}(2 l-t)\right) .
\end{aligned}
$$

In fact, Theorem 3.1 is true for a much wider class of functions $Q$ than the one given by (3.4). Similar to Theorem 2.2 in [26], the next theorem is immediate from Theorem 3.1 and equality $\widehat{U} A \widehat{U}=A^{*}((\widehat{U} f)(x)=\overline{f(l-x)})$.

Theorem 3.2 Suppose $S \in\left\{L_{p}^{2}(0, l), L_{p}^{2}(0, l)\right\}$ satisfies the operator identity $A S-S A^{*}=i \int_{0}^{l}\left(\Phi_{1}(x)+\widehat{\Phi}_{1}(t)\right) \cdot d t$, where $\Phi_{1}(x)$ and $\widehat{\Phi}_{1}(t)$ are $p \times p$ matrix functions with the entries from $L^{2}(0, l)$. Then $S$ is an operator with a $D$ difference kernel, i.e., the operator of the form (1.1), (1.2), and $s(u, 0)=$ $D^{-1} \Phi_{1}(u), s(0, u)=-D^{-1} \widehat{\Phi}_{1}(u)$. Moreover, when (3.1) holds, that is, $\widehat{\Phi}_{1}(t)=\Phi_{1}(t)^{*}$ we have

$$
s(x, t)=-D^{-1} s(t, x)^{*} D, \quad S=S^{*} .
$$




\section{Positive operators $S$ and an inverse prob- lem for canonical system}

Operators with $D$-difference kernels are essential for the construction of solutions of an inverse problem for an important subclass of canonical systems $[21,28,29]$. Canonical system is a system of the form

$$
\frac{d}{d x} w(x, \lambda)=i \lambda J H(x) w(x, \lambda), \quad H(x) \geq 0, \quad J=\left[\begin{array}{cc}
0 & I_{p} \\
I_{p} & 0
\end{array}\right],
$$

where the Hamiltonian $H$ is a $m \times m(m=2 p)$ locally summable matrix function. A Weyl function of the canonical system on the semi-axis $x \geq 0$ is a $p \times p$ matrix function $\varphi(\lambda)$, which is analytic in $\mathbb{C}_{+}$and satisfies the condition [29]

$$
\int_{0}^{\infty}\left[\begin{array}{ll}
I_{p} & i \varphi(\lambda)^{*}
\end{array}\right] w(x, \lambda)^{*} H(x) w(x, \lambda)\left[\begin{array}{c}
I_{p} \\
-i \varphi(\lambda)
\end{array}\right] d x<\infty, \quad \lambda \in \mathbb{C}_{+} .
$$

The corresponding inverse problem is the problem to recover $H$ or, equivalently, canonical system from the Weyl function. In the case of rational Weyl matrix functions several inverse problems were solved explicitly using a GBDT version of the Bäcklund-Darboux transformation [5, 10, 11, 20, 22]. (See $[3,7,15,19,22,31]$ and references therein for various versions of the Bäcklund-Darboux transformation and commutation methods.) However, taking into account that the positivity of operators $S$ and the application of the inversion formulas for semiseparable operators is of independent interest, we shall use a general scheme [25, 29] and its modification [21] for the inverse problem treated in this section. As a result of the application of the general scheme to rational matrix functions, semiseparable operators appear. Inverse problems for self-adjoint and skew-self-adjoint Dirac-type systems were studied using semiseparable operators in [1] and [6], respectively.

Consider rational Herglotz $p \times p$ matrix functions $\varphi$. The statement below is immediate from Theorem 5.2 [11].

Proposition 4.1 If $\varphi$ is a rational matrix function such that

$$
\lim _{\lambda \rightarrow \infty} \varphi(\lambda)=\frac{i}{2} D, \quad \Im \varphi(\lambda) \geq 0 \quad\left(\lambda \in \mathbb{C}_{+}\right),
$$


then $\varphi$ admits a representation (i.e., realization in terms of control theory)

$$
\varphi(\lambda)=\frac{i}{2} D+\Theta_{1}^{*}\left(\beta-\lambda I_{n}\right)^{-1} \Theta_{2}
$$

where $\Theta_{1}$ and $\Theta_{2}$ are $n \times p$ matrix functions, $n$ is some positive integer number, and $n \times n$ matrix $\beta$ satisfies the matrix identity

$$
\beta^{*}-\beta=i\left(\Theta_{2}-\Theta_{1}\right) D^{-1}\left(\Theta_{2}-\Theta_{1}\right)^{*} .
$$

Dirac systems and Weyl matrix functions $\widetilde{\varphi}$, which have the form $\widetilde{\varphi}=$ $2 D^{-\frac{1}{2}} \varphi D^{-\frac{1}{2}}$, were studied in [10]. The next proposition follows from the Step 1 of the proof of Theorem 4.3 [10] (see also [11]).

Proposition 4.2 Let relations (4.4) and (4.5) hold. Then $\Im \varphi(\lambda)>0(\lambda \in$ $\left.\mathbb{C}_{+}\right)$and $\varphi$ admits Herglotz representation

$$
\varphi(\lambda)=\nu+\int_{-\infty}^{\infty}\left(\frac{1}{z-\lambda}-\frac{z}{1+z^{2}}\right) d \tau(z) \quad\left(\nu=\nu^{*}\right)
$$

where

$$
\tau(z)=\int_{0}^{z} \rho(t) d t+\sum_{z_{k}<z} \nu_{k}
$$

numbers $z_{1}<z_{2}<\ldots$ are the real eigenvalues of $\beta$,

$$
\nu_{k}=\operatorname{res}_{z=z_{k}} \Theta_{2}^{*}\left(z I_{n}-\beta\right)^{-1} \Theta_{2} \geq 0,
$$

and $\rho$ is $p \times p$ rational matrix function:

$$
\rho(t)=\frac{1}{2 \pi} \zeta(t)^{*} D \zeta(t) \geq 0, \quad \zeta(t):=I_{p}-i D^{-1}\left(\Theta_{2}-\Theta_{1}\right)^{*}\left(t I_{n}-\beta\right)^{-1} \Theta_{2} .
$$

It is easy to see from (3.2) that

$$
(I-z A)^{-1} \Phi_{2}=e^{i z x D}, \quad \Phi_{2}^{*}\left(I-z A^{*}\right)^{-1} f=\int_{0}^{l} e^{-i z x D} f(x) d x \quad\left(f \in L_{p}^{2}(0, l)\right) .
$$

By (4.7)-(4.10) the right-hand side of the equality

$$
S:=\int_{-\infty}^{\infty}(I-z A)^{-1} \Phi_{2} d \tau(z) \Phi_{2}^{*}\left(I-z A^{*}\right)^{-1}
$$


weakly converges, and so the equality defines an operator $S$. Moreover, it is easy to see that the inequalities

$$
c(f, f)_{L^{2}}>(S f, f)_{L^{2}}>0
$$

hold for some fixed $c>0$ and arbitrary $f \neq 0$. (Here $(\cdot, \cdot)_{L^{2}}$ denotes the scalar product in $L_{p}^{2}(0, l)$.) Thus, $S$ is a bounded and positive operator. We shall show that operators $S$ belong to a subclass of operators of the form (2.1), (2.2).

Theorem 4.3 Let the matrix identity (4.5) hold. Then the operator $S$ given by (2.1) and (2.2) is positive and boundedly invertible.

Proof. The theorem is obtained by proving that $S$ of the form (2.1), (2.2) admits representation (4.11).

First, consider $S$ given by (4.11). It can be calculated directly (see also Section 1.1 in [27]) that this operator $S$ satisfies the operator identity (3.1), where $\Pi=\left[\begin{array}{ll}\Phi_{1} & \Phi_{2}\end{array}\right]$ and

$$
\Phi_{1}=i\left(\nu-\int_{-\infty}^{\infty}\left(A(I-z A)^{-1}+\frac{z}{1+z^{2}} I\right) \Phi_{2} d \tau(z)\right) .
$$

Here the operator $\Phi_{1}$ is an operator of multiplication by the matrix function, which we denote by $\Phi_{1}(x)$. From the identity (3.1) and Theorem 3.2 it follows that $S$ is an operator with a $D$-difference kernel $s(x, t)=\left\{s_{i j}\left(d_{i} x-d_{j} t\right)\right\}_{i, j=1}^{p}$ and $s(x, 0)=D^{-1} \Phi_{1}(x)$. Introduce $S=S_{l}$ and $\Phi_{1}=\Phi_{1, l}$ by (4.11) and (4.13), respectively, for all $0<l<\infty$. Then the kernel $s(x)$ of the integral operators $S_{l}$ is determined on $\mathbb{R}$ by the equalities

$$
s_{i j}(x)=d_{i}^{-1}\left(\Phi_{1}\right)_{i j}\left(x / d_{i}\right) \quad(x>0), \quad s_{i j}(-x)=-\frac{d_{j}}{d_{i}} \overline{s_{j i}(x)} .
$$

For $\varphi$ satisfying (4.6), according to Statement 3 in [21], after the corresponding change of notations we get

$$
\varphi(\lambda)=\lambda \int_{0}^{\infty} s(x, 0)^{*} e^{i \lambda x D} d x D^{2}=\lambda \int_{0}^{\infty} e^{i \lambda x} s(x)^{*} d x D .
$$

Note that in view of formula (4.13) and Proposition 4.2 we can present $s$ as a sum $s(x)=s_{1}(x)+s_{2}(x)$, where the entries of $s_{1}$ are bounded and the 
entries of $s_{2}$ belong $L^{2}(0, \infty)$. Finally, we apply Fourier transform to derive from (4.15) the equality

$$
e^{-\eta x} s(x)^{*}=\frac{1}{2 \pi} \text { l.i.m. }{ }_{a \rightarrow \infty} \int_{-a}^{a} e^{-i \xi x} \lambda^{-1} \varphi(\lambda) D^{-1} d \xi \quad(\lambda=\xi+i \eta, \quad \eta>0),
$$

the limit l.i.m. being the limit in $L^{2}(0, l)(0<l<\infty)$. Using (4.4) and (4.16), we obtain

$$
e^{-\eta x} s(x)^{*}=\frac{1}{2 \pi} \text { l.i.m. }_{a \rightarrow \infty} \int_{\Gamma_{a}} e^{-i \xi x} \lambda^{-1} \varphi(\lambda) D^{-1} d \xi \quad(\lambda=\xi+i \eta, \quad \eta>0),
$$

where $\Gamma_{a}$ is a clockwise oriented contour:

$$
\Gamma_{a}=[-a, a] \cup\{\xi:|\xi|=a, \Im \xi<0\} .
$$

It is easy to see that

$$
\frac{1}{2 \pi} \text { l.i.m. } a \rightarrow \infty \int_{\Gamma_{a}} e^{-i \xi x} \lambda^{-1} d \xi=-i e^{-\eta x} .
$$

According to $(4.5)$ we have $\sigma(\beta) \subset \overline{\mathbb{C}_{-}}$, where $\sigma$ is spectrum. Similar to [6] we turn to zero $\varepsilon$ in the equality $\lambda^{-1}\left(\beta_{\varepsilon}-\lambda I_{n}\right)^{-1}=\beta_{\varepsilon}^{-1}\left(\lambda^{-1} I_{n}+\left(\beta_{\varepsilon}-\lambda I_{n}\right)^{-1}\right)$, where $\operatorname{det} \beta_{\varepsilon} \neq 0,\left\|\beta-\beta_{\varepsilon}\right\|<\varepsilon$, and thus obtain

$$
\frac{1}{2 \pi} \text { l.i.m. }{ }_{a \rightarrow \infty} \int_{\Gamma_{a}} e^{-i \xi x} \lambda^{-1}\left(\beta-\lambda I_{n}\right)^{-1} d \xi=e^{-\eta x} \int_{0}^{x} \exp (-i u \beta) d u .
$$

Here we take into account that, when the spectrum of some matrix $\mathcal{K}$ is situated inside the anti-clockwise oriented contour $\Gamma$ we have

$$
\frac{1}{2 \pi i} \int_{\Gamma} e^{-i \lambda x}\left(\lambda I_{n}-\mathcal{K}\right)^{-1} d \lambda=\exp (-i x \mathcal{K})
$$

By (4.4) and (4.17)-(4.19) we get

$$
s(x)=\frac{1}{2} I_{p}+D^{-1} \Theta_{2}^{*} \int_{0}^{x} \exp \left(i u \beta^{*}\right) d u \Theta_{1} \quad(x>0) .
$$

It follows from (4.14) that $s(x)=-D^{-1} s(-x)^{*} D(x<0)$, and so according to $(4.20) s(x)$ is continuously differentiable for $x \neq 0$. As the functions $s_{i j}(x)$ 
are continuous at $x=0$ for $i \neq j$, and $s_{i i}(+0)-s_{i i}(-0)=1$, formulas $(1.1)$ and (1.2) imply (2.1), where $k(x)=D\left(\frac{d}{d x} s\right)(x)$. Therefore we have

$$
k(x)=\Theta_{2}^{*} \exp \left(i x \beta^{*}\right) \Theta_{1} \quad(x>0), \quad k(x)=k(-x)^{*} .
$$

Now, note that equalities (2.2) and (4.21) coincide. In other words, the operator $S$, which is considered in the theorem, admits representation (4.11). Hence, by (4.12) this operator is bounded and positive, and so in view of (2.1) and (2.2) it is also boundedly invertible.

The matrix function $\tau$ of the form (4.7)-(4.9) and the $S$-node given by (3.2), (4.11), and (4.13) satisfy conditions of Theorem 2.4 [29], p. 57. Therefore $\varphi(\lambda)$ given by (4.6) can be presented as a linear-fractional transformation

$$
\varphi(\lambda)=i\left(\mathcal{W}_{11}(\lambda) R_{1}(\lambda)+\mathcal{W}_{12}(\lambda) R_{2}(\lambda)\right)\left(\mathcal{W}_{21}(\lambda) R_{1}(\lambda)+\mathcal{W}_{22}(\lambda) R_{2}(\lambda)\right)^{-1}
$$

where $\mathcal{W}_{i j}(\lambda)$ are $p \times p$ blocks of the matrix function $\mathcal{W}$,

$$
\mathcal{W}(\lambda):=W(l, \bar{\lambda})^{*}, \quad W(l, \lambda)=I_{2 p}+i \lambda J \Pi^{*} S^{-1}(I-\lambda A)^{-1} \Pi,
$$

and $R_{1}(\lambda)=R_{1}(l, \lambda), R_{2}(\lambda)=R_{2}(l, \lambda)$ is a pair of $p \times p$ matrix functions, which are meromorphic in $\mathbb{C}_{+}$and have property- $J$, that is,

$$
R_{1}(\lambda)^{*} R_{1}(\lambda)+R_{2}(\lambda)^{*} R_{2}(\lambda)>0, \quad\left[\begin{array}{ll}
R_{1}(\lambda)^{*} & R_{2}(\lambda)^{*}
\end{array}\right] J\left[\begin{array}{l}
R_{1}(\lambda) \\
R_{2}(\lambda)
\end{array}\right] \geq 0 .
$$

It is easy to see from (4.23) that $\lim _{l \rightarrow+0} W(l, \lambda)=I_{2 p}$ and thus we put $W(0, \lambda)=I_{2 p}$. Now, by Theorem 2.1 from [29], p.54 the matrix function $W$ satisfies for $x \geq 0$ the equation

$$
W(x, \lambda)=I_{2 p}+i \lambda J \int_{0}^{x}\left(d B_{1}(r)\right) W(r, \lambda), \quad B_{1}(r):=\Pi_{r}^{*} S_{r}^{-1} \Pi_{r},
$$

where $S_{r} \in\left\{L_{p}^{2}(0, r), L_{p}^{2}(0, r)\right\}, \Pi_{r} \in\left\{\mathbb{C}^{2 p}, L_{p}^{2}(0, r)\right\}$. As the operators $S_{r}$ $(0<r \leq l<\infty)$ are invertible, the operators $S_{l}$ admit triangular factorisation (see [14], p. 184). It follows that $B_{1}$ is differentiable, and we rewrite (4.25) as the canonical system

$$
\begin{aligned}
& \frac{d}{d x} W(x, \lambda)=i \lambda J H(x) W(x, \lambda), \\
& H(x):=\frac{d}{d x}\left(\Pi_{x}^{*} S_{x}^{-1} \Pi_{x}\right) .
\end{aligned}
$$


Moreover, in view of Remark 2.4 the kernel $T_{r}(x, t)$ of the integral operator $S_{r}^{-1}$ is continuous with respect to $x, t, r$ excluding the lines $d_{i} x=d_{j} t$. Therefore, for $d_{i} x \neq d_{j} r(1 \leq i, j \leq p)$ similar to the continuous kernels ([14], p.186) we have

$$
k(x, r)+T_{r}(x, r)+\int_{0}^{r} k(x, u) T_{r}(u, r) d u=0, \quad x \leq r \leq l .
$$

Introduce an upper triangular operator

$$
V_{+}=I+\int_{x}^{l} T_{r}(x, r) \cdot d r \in\left\{L_{p}^{2}(0, l)\right\} .
$$

According to (4.28) and (4.29) the operator $S_{l} V_{+}$is a lower triangular operator. Hence, the operator $V_{+}^{*} S_{l} V_{+}$is a lower triangular operator. On the other hand $V_{+}^{*} S_{l} V_{+}$is selfadjoint, and so the integral part of $V_{+}^{*} S_{l} V_{+}$equals zero, i.e., $V_{+}^{*} S_{l} V_{+}=I$ or equivalently

$$
S_{l}^{-1}=V_{+} V_{+}^{*}, \quad V_{+, l}^{*}=V_{+}^{*}=I+\int_{0}^{x} T_{x}(x, r) \cdot d r .
$$

In the second equality above we used formula (4.29) and relation $T_{x}(r, x)^{*}=$ $T_{x}(x, r)(x \geq r)$.

Theorem 4.4 Let $\varphi$ be a rational function, which satisfies (4.3). Then $\varphi$ is a Weyl function of the canonical system (4.26), where the Hamiltonian $H$ has the form

$$
H(x)=\gamma(x)^{*} \gamma(x), \quad \gamma(x)=\left(V_{+}^{*}\left[\begin{array}{ll}
\Phi_{1} & \Phi_{2}
\end{array}\right]\right)(x) \quad(x \leq l<\infty),
$$

and the operator $V_{+}^{*}$ is given by (4.30) and is applied columnwise to the matrix functions $\Phi_{1}(x)=\left\{d_{i} s_{i j}\left(d_{i} x\right)\right\}_{i, j=1}^{p}$ and $\Phi_{2} \equiv I_{p}$. The matrix function $s(x)$ is given by (4.20) and the matrix function $T_{x}(x, r)$ in (4.30) is given in Remark 2.4.

Pr o of. It follows from (4.26) that

$$
\begin{aligned}
& \frac{d}{d x}\left(W(x, \bar{\lambda})^{*} J W(x, \lambda)\right)=0, \\
& \frac{d}{d x}\left(W(x, \lambda)^{*} J W(x, \lambda)\right)=i(\lambda-\bar{\lambda}) W(x, \lambda)^{*} H(x) W(x, \lambda) .
\end{aligned}
$$


In view of (4.33) we obtain

$$
\int_{0}^{l} W(x, \lambda)^{*} H(x) W(x, \lambda) d x=i(\bar{\lambda}-\lambda)^{-1}\left(W(l, \lambda)^{*} J W(l, \lambda)-J\right) .
$$

Note also that according to (4.32) the equality $W(l, \bar{\lambda})^{*} J W(l, \lambda)=J$ holds, or equivalently

$$
W(l, \bar{\lambda})^{*}=J W(l, \lambda)^{-1} J .
$$

By Proposition $4.1 \varphi$ admits representation (4.4) and identity (4.5) is valid. So, by Proposition $4.2 \varphi$ admits Herglotz representation, where the matrix function $\tau(t)$ has the form (4.7)-(4.9). Hence, as it was shown above, the representation (4.22) of $\varphi$, where $\mathcal{W}$ is expressed via the matrizant $W(l, \lambda)$ and the pair $R_{1}, R_{2}$ satisfies (4.24), is also true. Using (4.35), we rewrite (4.22) in the form

$$
\left[\begin{array}{c}
I_{p} \\
-i \varphi(\lambda)
\end{array}\right]=W(l, \lambda)^{-1} J\left[\begin{array}{l}
R_{1}(\lambda) \\
R_{2}(\lambda)
\end{array}\right]\left(\mathcal{W}_{21}(\lambda) R_{1}(\lambda)+\mathcal{W}_{22}(\lambda) R_{2}(\lambda)\right)^{-1}
$$

Taking into account (4.24), (4.34), and (4.36) we derive

$$
\begin{aligned}
& \int_{0}^{l}\left[\begin{array}{ll}
I_{p} & i \varphi(\lambda)^{*}
\end{array}\right] W(x, \lambda)^{*} H(x) W(x, \lambda)\left[\begin{array}{c}
I_{p} \\
-i \varphi(\lambda)
\end{array}\right] d x \leq i(\lambda-\bar{\lambda})^{-1} \\
& \times\left[\begin{array}{ll}
I_{p} & i \varphi(\lambda)^{*}
\end{array}\right] J\left[\begin{array}{c}
I_{p} \\
-i \varphi(\lambda)
\end{array}\right], \quad \lambda \in \mathbb{C}_{+}
\end{aligned}
$$

As the right-hand side in the inequality (4.37) does not depend on $l$ we can substitute $\infty$ instead of the limit $l$ of integration in the left-hand side. Hence $\varphi$ is a Weyl function of the constructed system.

According to the second relation in (4.30) we obtain $\left(V_{+, l}^{*} f\right)(x)=\left(V_{+, x}^{*} \widetilde{f}\right)(x)$ for $x \leq l$, where $\tilde{f}$ is the restriction of $f$ on the interval $[0, x]$. Therefore, relations (4.27) and (4.30) imply (4.31).

Corollary 4.5 Let the conditions of Theorem 4.4 hold and let $\operatorname{det} \beta \neq 0$. Then we have

$$
\gamma(x)=\left(V_{+}^{*}\left[\frac{1}{2} D+i \Theta_{2}^{*}\left(\beta^{*}\right)^{-1} \Theta_{1} \quad I_{p}\right]\right)(x)-i\left[\begin{array}{ll}
\gamma_{0}(x) & 0
\end{array}\right],
$$


where the $s$-th row of $\gamma_{0}(p \geq s \geq 1)$ is given by the equality

$$
\begin{aligned}
& e_{s} \gamma_{0}(x)=e_{s}\left(\Theta_{2}^{*} e^{i d_{s} x \beta^{*}}+\left[\begin{array}{ll}
\Theta_{2}^{*} & \Theta_{1}^{*}
\end{array}\right] e^{d_{s} x \mathcal{A}} U\left(d_{s} x\right)\right. \\
& \left.\times\left(P^{\times} U\left(d_{1} x\right)^{-1}-U\left(d_{s} x\right)^{-1}+I_{2 n}-P^{\times}\right)\left[\begin{array}{c}
I_{p} \\
0
\end{array}\right]\right)\left(\beta^{*}\right)^{-1} \Theta_{1},
\end{aligned}
$$

$U$ in (4.39) is defined by (2.24) after substitution $l=x$, and $P^{\times}$is defined by (2.16) after substitution $a=d_{1} l=d_{1} x$.

Pr o of. By (1.2), (3.2), and (4.20) the equality

$$
e_{s}\left[\Phi_{1}(x) \quad \Phi_{2}\right]=e_{s}\left[\frac{1}{2} D+i \Theta_{2}^{*}\left(\beta^{*}\right)^{-1} \Theta_{1}-i \Theta_{2}^{*} e^{i d_{s} x \beta^{*}}\left(\beta^{*}\right)^{-1} \Theta_{1} \quad I_{p}\right]
$$

is true. Using (4.40) and the second equlity in (4.31) we obtain (4.38), where

$$
\gamma_{0}(x)=\left(V_{+}^{*}\left\{e_{s} \Theta_{2}^{*} e^{i d_{s} x \beta^{*}}\left(\beta^{*}\right)^{-1} \Theta_{1}\right\}_{s=1}^{p}\right)(x) .
$$

From (2.21) it follows that

$$
\Theta_{2}^{*} e^{i d_{s} x \beta^{*}}=\left[\begin{array}{ll}
\Theta_{2}^{*} & \Theta_{1}^{*}
\end{array}\right] e^{d_{s} x \mathcal{A}}\left[\begin{array}{c}
I_{p} \\
0
\end{array}\right] .
$$

According to the representation of $V_{+}^{*}$ in (4.30), Remark 2.4, formula (4.42) and second relation in (2.23) we get

$$
V_{+}^{*}\left\{e_{s} \Theta_{2}^{*} e^{i d_{s} x \beta^{*}}\right\}_{s=1}^{p}=\left\{e_{s} \Theta_{2}^{*} e^{i d_{s} x \beta^{*}}\right\}_{s=1}^{p}+\left\{\mathcal{F}_{s}(x) \mathcal{G}_{s}(x)\right\}_{s=1}^{p},
$$

where

$$
\begin{gathered}
\mathcal{F}_{s}(x)=e_{s}\left[\begin{array}{ll}
\Theta_{2}^{*} & \Theta_{1}^{*}
\end{array}\right] e^{d_{s} x \mathcal{A}} U\left(d_{s} x\right) \\
\mathcal{G}_{s}(x)=\left(\left(I_{2 n}-P^{\times}\right) \int_{0}^{d_{s} x} U(z)^{-1} e^{-z \mathcal{A}} Y(z) e^{z \mathcal{A}} d z\right. \\
\left.-P^{\times} \int_{d_{s} x}^{d_{1} x} U(z)^{-1} e^{-z \mathcal{A}} Y(z) e^{z \mathcal{A}} d z\right)\left[\begin{array}{c}
I_{p} \\
0
\end{array}\right] \\
Y(z)=\sum_{j: d_{j}>\widetilde{d}_{m}} \frac{1}{d_{j}}\left[\begin{array}{c}
-\Theta_{1} \\
\Theta_{2}
\end{array}\right] e_{j}^{*} e_{j}\left[\begin{array}{cc}
\Theta_{2}^{*} & \Theta_{1}^{*}
\end{array}\right]=Y_{m} \quad \text { for } \quad \widetilde{d}_{m} x \leq z \leq \widetilde{d}_{m-1} x .
\end{gathered}
$$


Taking into account (2.25) rewrite (4.45) in the form

$\mathcal{G}_{s}(x)=\left(\left(I_{2 n}-P^{\times}\right)\left(I_{2 n}-U\left(d_{s} x\right)^{-1}\right)+P^{\times}\left(U\left(d_{1} x\right)^{-1}-U\left(d_{s} x\right)^{-1}\right)\right)\left[\begin{array}{c}I_{p} \\ 0\end{array}\right]$

Finally, formulas (4.41), (4.43), (4.44), and (4.46) imply (4.39).

In view of Corollary 4.5, to recover $\gamma$ and Hamiltonian $H$ we need only to calculate the action of $V_{+}^{*}$ on constant vectors.

The matrix function $\gamma(x)$, which is recovered in Theorem 4.4, satisfies the equality

$$
\gamma(x) J \gamma(x)^{*} \equiv D
$$

Indeed, by (3.1), the first equality in (4.30), and the second equality in (4.31) we have

$$
V_{+}^{*} A\left(V_{+}^{*}\right)^{-1}-V_{+}^{-1} A^{*} V_{+}=i \gamma(x) J \int_{0}^{l} \gamma(t)^{*} \cdot d t
$$

As $V_{+}^{*} A\left(V_{+}^{*}\right)^{-1}$ is a lower triangular operator and $V_{+}^{-1} A^{*} V_{+}$is an upper triangular operator, we derive

$$
V_{+}^{*} A\left(V_{+}^{*}\right)^{-1}=i \gamma(x) J \int_{0}^{x} \gamma(t)^{*} \cdot d t .
$$

Rewrite (4.49) in terms of the kernels of the corresponding integral operators and put $t=x$ to get (4.47).

As it is stated in the proposition below, equality (4.47) means that we recover canonical systems from the subclass of systems with linear similar matrix functions $J H(x)$, though (differently from [29], p. 104) the kernel of $S^{-1}$ is not necessarily continuous.

Proposition 4.6 Let the conditions of Theorem 4.4 hold. Then JH(x) is similar to the matrix $\mathrm{JH}_{0}$, where

$$
H_{0}:=\left[\begin{array}{cc}
D & 0 \\
0 & 0
\end{array}\right]
$$

Pr o of. Fix $x \geq 0$ and denote by $X$ a $p \times 2 p$ matrix such that it has rank $p$ and satisfies the equality $X J \gamma(x)^{*}=0$. As the maximal $J$-nonnegative subspaces 
are $p$-dimensional, it easily follows from $\gamma(x) J \gamma(x)^{*}>0$ and $X J \gamma(x)^{*}=0$ that $X J X^{*}<0$. Then, we have

$$
\widetilde{X} J \widetilde{X}^{*}=-I_{p}, \quad \widetilde{X} J \gamma(x)^{*}=0 \quad \text { for } \quad \tilde{X}:=\left(-X J X^{*}\right)^{-\frac{1}{2}} X .
$$

Now, put

$$
L:=\left[\begin{array}{c}
D^{-\frac{1}{2}} \gamma(x) \\
\widetilde{X}
\end{array}\right] .
$$

By (4.47), (4.51), and (4.52) the equality

$$
L^{-1}=\left[\begin{array}{ll}
J \gamma(x)^{*} D^{-\frac{1}{2}} & -J \widetilde{X}^{*}
\end{array}\right]
$$

is true. According to (4.50), (4.52), and (4.53) we get $L^{-1} H_{0} L=J \gamma(x)^{*} \gamma(x)$. In view of (4.31) the last equality yields $L^{-1} H_{0} L=J H(x)$.

Acknowledgement. The work of A.L. Sakhnovich was supported by the Austrian Science Fund (FWF) under Grant no. Y330, and his visit to Mexico was supported by the PIFI grant P/CA-9 2007-14-17. A.L. Sakhnovich is grateful to the Autonomous University of Hidalgo for its hospitality.

\section{References}

[1] D. Alpay, I. Gohberg, L. Lerer, M.A. Kaashoek, and A.L. Sakhnovich, Krein systems, in: OT: Adv. Appl. 191, 2009, 19-36.

[2] H. Bart, I. Gohberg, and M.A. Kaashoek, Convolution equations and linear systems, IEOT 5 (1982), 283-340.

[3] P.A. Deift, Applications of a commutation formula, Duke Math. J. 45 (1978), 267-310.

[4] M.J. Corless and A.E. Frazho, Linear Systems and Control - An Operator Perspective, Marcel Dekker, New York, 2003.

[5] B. Fritzsche, B. Kirstein, and A.L. Sakhnovich, Completion problems and scattering problems for Dirac type differential equations with singularities, J. Math. Anal. Appl. 317 (2006), 510-525. 
[6] B. Fritzsche, B. Kirstein, and A.L. Sakhnovich, Semiseparable integral operators and explicit solution of an inverse problem for the skew-self-adjoint Dirac type system, arXiv:0904.2357

[7] F. Gesztesy and G. Teschl, On the double commutation method, Proc. Am. Math. Soc. 124:6 (1996), 1831-1840.

[8] I. Gohberg, S. Goldberg, and M.A. Kaashoek, Classes of Linear Operators, Volume I, Birkhäuser Verlag, Basel, 1990.

[9] I. Gohberg and M.A. Kaashoek, Time varying linear systems with boundary conditions and integral operators. I. The transfer operator and its properties, IEOT 7 (1984), 325-391.

[10] I. Gohberg, M.A. Kaashoek, and A.L. Sakhnovich, Canonical systems with rational spectral densities: explicit formulas and applications, Math. Nachr. 194 (1998), 93-125.

[11] I. Gohberg, M.A. Kaashoek, and A.L. Sakhnovich, Scattering problems for a canonical system with a pseudo-exponential potential, Asymptotic Analysis, 29:1 (2002), 1-38.

[12] I. Gohberg, M.A. Kaashoek and F. van Schagen, On inversion of convolution integral operators on a finite interval, OT: Adv. Appl. 147 (2004), Birkhäuser, Basel, 277-285.

[13] I.C. Gohberg and M.G. Krein, Systems of integral equations on a half line with kernels depending on the difference of arguments, Amer. Math. Soc. Transl. (2) 14 (1960), 217-287.

[14] I.Gohberg and M.G.Krein, Theory and applications of Volterra operators in Hilbert space, Nauka, Moscow, 1967. Translated in: Transl. of math. monographs 24, Providence, Rhode Island, 1970.

[15] C.H. Gu, H. Hu, and Z. Zhou, Darboux transformations in integrable systems, Springer Verlag, 2005. 
[16] M. Kac, On some connections between probability theory and differential and integral equations, Proc. Berkeley Sympos. Math. Statist. Probability, California Juli 31- August 12, 1950 (1951), 189-215.

[17] A. A. Karelin, Kh. Peres Lechuga, and A.A. Tarasenko, The Riemann problem and singular integral equations with coefficients generated by piecewise-constant functions. (Russian) Differ. Uravn. 44:9 (2008), 1182-1192.

[18] M.G. Krein, Integral equations on the half-line with a kernel depending on the difference of the arguments, (Russian) Uspehi Mat. Nauk 13:5(83) (1958), 3-120.

[19] V.B. Matveev and M.A. Salle, Darboux transformations and solitons, Springer Verlag, Berlin, 1991.

[20] R. Mennicken, A.L. Sakhnovich, and C. Tretter, Direct and inverse spectral problem for a system of differential equations depending rationally on the spectral parameter, Duke Math. J. 109:3 (2001), 413-449.

[21] A.L. Sakhnovich, Asymptotics of spectral functions of an S-node, Soviet Math. (Iz. VUZ) 32 (1988), 92-105.

[22] A.L. Sakhnovich, Iterated Bäcklund-Darboux transform for canonical systems, J. Functional Anal. 144 (1997), 359-370.

[23] A.L. Sakhnovich, Generalized Bäcklund-Darboux transformation: spectral properties and nonlinear equations, JMAA 262 (2001), 274306.

[24] L.A. Sakhnovich, Equations with a difference kernel on a finite interval, Russian Math. Surv. 35 (1980), 81-152.

[25] L.A. Sakhnovich, Factorisation problems and operator identities, Uspekhi Mat. Nauk 41 :1 (1986), 3-55; English transl. in Russian Math. Surveys 41 (1986), 1-64. 
[26] L.A. Sakhnovich, Integral equations with difference kernels on finite intervals, Operator Theory: Adv. Appl. 84, Birkhäuser, BaselBoston-Berlin, 1996.

[27] L.A. Sakhnovich, Interpolation theory and its applications, Mathematics and its Applications 428, Kluwer Academic Publishers, Dordrecht, 1997.

[28] L.A. Sakhnovich, On a class of canonical systems on half-axis, IEOT 31 (1998), 92-112.

[29] L.A. Sakhnovich, Spectral theory of canonical differential systems, method of operator identities, OT: Adv. Appl. 107, Birkhäuser Verlag, Basel-Boston, 1999.

[30] R. Vandebril, M. Van Barel, G. Golub, and N. Mastronardi, A bibliography on semiseparable matrices, Calcolo 42 (2005), 249-270.

[31] V.E.Zakharov and A.V.Mikhailov, On the integrability of classical spinor models in two-dimensional space-time, Comm. Math. Phys. 74 (1980), 21-40.

A.L. Sakhnovich, Fakultät für Mathematik, Universität Wien, Nordbergstrasse 15, A-1090 Wien, Austria e-mail: al_sakhnov@yahoo.com

A.A. Karelin, Universidad Autonoma del Estado de Hidalgo, Instituto de Ciencias Basicas e Ingenieria Centro de investigacin Avanzada en Ingeniera Industrial Carretera Pachuca-Tulancingo, Km. 4,5 Ciudad Universitaria, C.P. 42180,

Pachuca, Hidalgo, Mexico karelin@uaeh.edu.mx

J. Seck-Tuoh-Mora, Universidad Autonoma del Estado de Hidalgo, Instituto de Ciencias Basicas e Ingenieria Centro de investigacin Avanzada en Ingeniera Industrial 
Carretera Pachuca-Tulancingo, Km. 4,5 Ciudad Universitaria, C.P. 42180, Pachuca, Hidalgo, Mexico jseck@uaeh.edu.mx G. Perez-Lechuga, Universidad Autonoma del Estado de Hidalgo, Instituto de Ciencias Basicas e Ingenieria Centro de investigacin Avanzada en Ingeniera Industrial Carretera Pachuca-Tulancingo, Km. 4,5 Ciudad Universitaria, C.P. 42180, Pachuca, Hidalgo, Mexico glechuga2004@hotmail.com

M. Gonzalez-Hernandez, Universidad Autonoma del Estado de Hidalgo, Instituto de Ciencias Basicas e Ingenieria Centro de investigacin Avanzada en Ingeniera Industrial Carretera Pachuca-Tulancingo, Km. 4,5 Ciudad Universitaria, C.P. 42180, Pachuca, Hidalgo, Mexico mghdez@uaeh.edu.mx 